\title{
Pannexin 1 channels mediate 'find-me' signal release and membrane permeability during apoptosis
}

\author{
Faraaz B. Chekeni ${ }^{1,2}$, Michael R. Elliott ${ }^{1,3}$, Joanna K. Sandilos ${ }^{2}$, Scott F. Walk ${ }^{1,3}$, Jason \\ M. Kinchen ${ }^{1,3,4}$, Eduardo R. Lazarowski ${ }^{6}$, Allison J. Armstrong ${ }^{1,3}$, Silvia Penuela ${ }^{7}$, Dale \\ W. Laird7, Guy S. Salvesen ${ }^{8}$, Brant E. Isakson ${ }^{5}$, Douglas A. Bayliss ${ }^{2}$, and Kodi S. \\ Ravichandran $1,3,4$ \\ ${ }^{1}$ Beirne B. Carter Center for Immunology Research, University of Virginia, Charlottesville, \\ Virginia 22908, USA \\ 2 Department of Pharmacology, University of Virginia, Charlottesville, Virginia 22908, USA \\ 3 The Center for Cell Clearance, University of Virginia, Charlottesville, Virginia 22908, USA \\ ${ }^{4}$ Department of Microbiology, University of Virginia, Charlottesville, Virginia 22908, USA \\ ${ }^{5}$ Cardiovascular Research Center and the Department of Molecular Physiology and Biological \\ Physics, University of Virginia, Charlottesville, Virginia 22908, USA \\ ${ }^{6}$ Department of Pharmacology, University of North Carolina, Chapel Hill, North Carolina 27599, \\ USA \\ 7 Department of Anatomy and Cell Biology, University of Western Ontario, London, Ontario N6A \\ $5 \mathrm{C} 1$, Canada \\ ${ }^{8}$ Program in Apoptosis and Cell Death Research, The Burnham Institute for Medical Research, \\ La Jolla, California 92037, USA
}

\begin{abstract}
Apoptotic cells release 'find-me' signals at the earliest stages of death to recruit phagocytes ${ }^{1}$. The nucleotides ATP and UTP represent one class of find-me signals ${ }^{2}$, but their mechanism of release is not known. Here, we identify the plasma membrane channel pannexin 1 (PANX1) as a mediator of find-me signal/nucleotide release from apoptotic cells. Pharmacological inhibition and siRNAmediated knockdown of PANX1 led to decreased nucleotide release and monocyte recruitment by apoptotic cells. Conversely, PANX1 over-expression enhanced nucleotide release from apoptotic cells and phagocyte recruitment. Patch-clamp recordings showed that PANX1 was basally inactive, and that induction of PANX1 currents occurred only during apoptosis. Mechanistically,
\end{abstract}

Correspondence and requests for materials should be addressed to K.S.R. (Ravi@virgina.edu).

Full Methods and any associated references are available in the online version of the paper at www.nature.com/nature. Supplementary Information is linked to the online version of the paper at www.nature.com/nature.

Author Contributions F.B.C. designed, performed, and analysed most of the experiments in this study with input from K.S.R. M.R.E. performed the migration assays, air pouch experiments, $\mathrm{qPCR}$, and flow cytometry, and provided advice and help on many aspects of this work. J.K.S. performed and analysed the patch-clamp studies with input from D.A.B. S.F.W. generated the PANX1 cleavage-site mutants. J.M.K. conducted phagocytosis assays and aided with flow cytometry of monomeric cyanine dye uptake by transiently transfected cells. Quantification of UTP in supernatants was performed by E.R.L. A.J.A. demonstrated pannexin 1 cleavage in apoptotic thymocytes. S.P., D.W.L. and G.S.S. provided key reagents and intellectual input. B.E.I. performed the scrape assay, and provided intellectual input on these studies. K.S.R. provided overall coordination with respect to conception, design, and supervision of the study. F.B.C. and K.S.R. wrote the manuscript with comments from co-authors.

Author Information Reprints and permissions information is available at www.nature.com/reprints. The authors declare no competing financial interests. Readers are welcome to comment on the online version of this article at www.nature.com/nature. 
PANX1 itself was a target of effector caspases (caspases 3 and 7), and a specific caspase-cleavage site within PANX1 was essential for PANX1 function during apoptosis. Expression of truncated PANX1 (at the putative caspase cleavage site) resulted in a constitutively open channel. PANX1 was also important for the 'selective' plasma membrane permeability of early apoptotic cells to specific dyes ${ }^{3}$. Collectively, these data identify PANX1 as a plasma membrane channel mediating the regulated release of find-me signals and selective plasma membrane permeability during apoptosis, and a new mechanism of PANX1 activation by caspases.

Previous studies have shown that ATP and UTP are released from intact apoptotic cells, with no gross leakage of cellular contents ${ }^{2,4}$. We initially considered three potential mechanisms for regulated nucleotide release from early-stage apoptotic cells: exocytosis, blebbing or passage via a plasma membrane channel. Treatment of cells undergoing apoptosis with the drug brefeldin A (blocking exocytosis ${ }^{5}$ ) or Y27632 (inhibiting the Rho kinase ROCK critical for membrane blebbing ${ }^{6,7}$ ) did not affect ATP release (data not shown). Therefore, we focused on potential channel(s) that might mediate nucleotide release during apoptosis.

Among the channels suggested to conduct ATP across the plasma membrane are the connexin and pannexin family channels ${ }^{8-10}$. Connexins form gap junctions between cells, mediating intercellular communication ${ }^{8,10}$, whereas pannexin channels are thought to function primarily in conducting intracellular molecules to the extracellular space ${ }^{8,9}$. Initially, we used pharmacological inhibitors known to inhibit these channels ${ }^{11}$. The gap junction inhibitors 18-alpha-glycyrrhetinic acid (18AGA) and flufenamic acid (FFA) had no detectable effect on apoptosis-induced ATP release (Supplementary Fig. 1a); however, these drugs were effective in blocking connexin-mediated intercellular communication in a HeLacell-based scrape assay (Supplementary Fig. 1b). The inhibitor carbenoxolone (CBX), which targets connexin and pannexin channels, inhibited ATP release during apoptosis

(Supplementary Fig. 1a). Interference by CBX in the ATP quantification assay was ruled out (Supplementary Fig. 2a), and CBX blocked ATP release at all time points assessed, ruling out changes in the kinetics of release (Fig. 1a). Although CBX-mediated inhibition of ATP release was comparable to that seen with zVAD $(\mathrm{OMe})$-fmk (zVAD; cell-permeable pancaspase inhibitor that blocks apoptosis and ATP release from apoptotic cells ${ }^{2}$ ), CBX did not inhibit apoptosis, based on caspase activation ${ }^{12}$ and 'eat-me' signal exposure (Supplementary Fig. 2b, c). CBX also inhibited UTP release after apoptosis induction (Supplementary Fig. 4a), and the ability of apoptotic cell supernatants to attract THP-1 monocytes (Supplementary Fig. 4b, c). The higher CBX concentration needed for maximum inhibition of ATP release from apoptotic cells ( $>100 \mu \mathrm{M})$ is likely due to binding of CBX by albumin (BSA) in the medium, as lower CBX concentrations were sufficient in lower BSA concentrations (Supplementary Fig. 3a, b). Probenecid, an inhibitor considered more specific for pannexins over connexins ${ }^{13}$, also blocked ATP release from apoptotic cells (Fig. 1a). While drug studies have their limitations, the profile of inhibition (sensitivity to CBX and probenecid, but not 18AGA and FFA), suggested a role for pannexin channels ${ }^{11,14}$, which we pursued further.

Among the three pannexins in humans (pannexin 1, 2 and 3) ${ }^{15,16}$, pannexin 1 (PANX1) was the most abundant in the human Jurkat T cells used above (Fig. 1b). siRNA-mediated knockdown of PANX1, followed by induction of apoptosis reduced ATP and UTP release compared to control (47\% and 68\% decrease in ATP and UTP, respectively; Fig. 1c-e and Supplementary Fig. 5a, b). The residual ATP release was likely due to incomplete knockdown of PANX1 (Fig. 1c and Supplementary Fig. 5a), and was inhibitable by CBX and zVAD (Supplementary Fig. 5c). Importantly, no morphological differences were seen between control- and PANXI-siRNA-transfected cells at the time of apoptosis induction 
(data not shown). Total cellular ATP content was also comparable (Supplementary Fig. 5e), ruling this out as a cause for reduced ATP release. PANX1 knockdown had no effect on apoptosis progression, assessed by apoptotic caspase activity and by annexin $\mathrm{V}$ staining (Fig. 1d and Supplementary Fig. 5d). Consistent with decreased nucleotide find-me signal release, apoptotic cell supernatants from PANX1-siRNA-transfected cells also recruited fewer monocytes in vitro (Fig. 1f). To address chemotactic activity in vivo, we used the dorsal air pouch model that we had used previously ${ }^{2}$; cell supernatants were injected into a sterile air pouch, and leukocytes attracted to the pouch were analysed after $24 \mathrm{~h}$ (Fig. 1g). Supernatants from apoptotic PANX1 knockdown cells recruited fewer leukocytes into the pouch than supernatants from control-siRNA-transfected cells (Fig. 1g). Collectively, the PANX1 knockdown studies and pharmacological inhibition by CBX and probenecid supported a role for PANX1 in the release of the find-me signals ATP and UTP from apoptotic cells.

We also generated Jurkat cells stably overexpressing Flag-tagged, full-length PANX1 (426 amino acids ${ }^{11}$; denoted PANX1-Flag) (Fig. 2a). Compared to control-vector-transfected cells, PANX1-Flag cells released more ATP and UTP upon induction of apoptosis (Fig. 2b, c), and their apoptotic cell supernatants recruited more monocytes (Fig. 2d). Even with overexpression of PANX1, the enhanced release of nucleotides was still regulated by the induction of apoptosis and sensitive to $\mathrm{zVAD}$ and CBX (Fig. 2b and data not shown). Thus, both siRNA-mediated knockdown of PANX1 ('loss of function') and overexpression of PANX1 ('gain of function') directly correlate with apoptosis-induced nucleotide release and find-me-signal-mediated monocyte recruitment.

PANX1 can conduct molecules up to $1 \mathrm{kDa}$ in size across the plasma membrane (ATP and UTP are 507 and 484 daltons, respectively $)^{8,9}$, including the monomeric cyanine dye YOPRO-1 (ref. ${ }^{17}$ ). Monomeric cyanine dyes are marketed as apoptotic cell indicators, but the mechanism by which apoptotic cells take up these dyes is not known ${ }^{3}$. We asked whether PANX1 mediates the entry of the dyes YO-PRO-1 and TO-PRO-3 (629 and 671 daltons, respectively) during apoptosis. Interestingly, the uptake of YO-PRO-1 and TO-PRO-3 by apoptotic Jurkat cells was largely PANX1-dependent. This PANX1-dependent membrane permeability was selective, as these early-apoptotic cells did not take up the dye propidium iodide (which marks necrotic cells; data not shown). PANX1 knockdown (Fig. 2e) and treatment with CBX (Supplementary Fig. 6a) significantly reduced YO-PRO-1 uptake, whereas transient overexpression of PANX1 enhanced TO-PRO-3 uptake (Supplementary Fig. 6b). These data suggest an essential role for PANX1 in the release of the find-me signals ATP and UTP, and in the selective plasma membrane permeability that has long been considered a hallmark of early-stage apoptotic cells ${ }^{3}$.

We demonstrated previously that apoptotic primary thymocytes release nucleotide find-me signals, which is critical for proper phagocytic clearance of dying thymocytes in $v_{i v o}{ }^{2}$. Therefore, we asked whether thymocytes depend on PANX1 for increased plasma membrane permeability during apoptosis. Apoptotic thymocytes took up YO-PRO-1, and treatment with CBX blocked this uptake (similar to the caspase inhibitor zVAD) (Fig. 2f). However, unlike zVAD, CBX did not inhibit apoptosis, assessed by phosphatidylserine exposure (Fig. 2f).

We next asked whether apoptosis-induced membrane permeability could be directly correlated with PANX1-dependent plasma membrane currents. We measured channel activity at the single-cell level via whole-cell patch-clamp ${ }^{18,19}$, and made several striking observations. First, CBX-sensitive currents were detected only in apoptotic cells. In bulk suspension, where cells undergo apoptosis at different rates ${ }^{20}$, PANX1 currents were only detectable in cells that were beginning to bleb (a morphological characteristic of apoptosis; 
denoted 'dying' cells), but not in cells that appeared 'live' (Fig. 3a-c). Second, the apoptosis-induced currents were unaffected by $100 \mu \mathrm{M}$ FFA, but were rapidly inhibited by $100 \mu \mathrm{M}$ CBX (Fig. 3b, d and Supplementary Figs 7a, b and 8a-d). This rapid inhibition of the current flux (Fig. 3b and Supplementary Fig. 8d) suggested that CBX acts directly on the channel responsible for the increased membrane permeability. Third, apoptosis-induced currents were much larger in cells stably over-expressing PANX1-Flag (Fig. 3c) and markedly reduced in cells with PANX1 knockdown (Fig. 3e). Importantly, aside from the magnitude, the voltage-dependent properties of the apoptosis-induced CBX-sensitive current were essentially identical in control cells, PANX1-Flag overexpressing cells, and PANX1 knockdown cells (Fig. 3d and Supplementary Fig. 8c). Moreover, the $\mathrm{IC}_{50}$ values for CBX and probenecid, using wild-type Jurkat cells, roughly correspond to concentrations published in the literature (Supplementary Fig. 9) ${ }^{11}$. Collectively, these patch-clamp studies demonstrate that, at the single-cell level, the magnitude of the membrane permeability induced during apoptosis strongly correlates with the level of PANX1 expression.

We next investigated apoptosis-induced, post-translational modification(s) of PANX1 that might regulate channel activity. Interestingly, detection of endogenous PANX1 in apoptotic Jurkat cells by immunoblotting decreased in a time-dependent manner, temporally correlating with procaspase 3 activation (Fig. 4a). The caspase inhibitor zVAD 'restored' the PANX1 band, whereas CBX did not (although both zVAD and CBX inhibit the apoptosisinduced nucleotide release) (Fig. 4a and Supplementary Fig. 10a). Loss of Panx1 immunoreactivity, and its restoration by zVAD, was also observed in apoptotic primary murine thymocytes (Fig. 4b). This suggested an intriguing possibility that PANX1 itself might be a target of caspase-mediated cleavage. Although another type of modification could have caused the loss of immunoreactivity to this carboxy-terminal antibody ${ }^{21}$ (CT Ab; Fig. 4c), this was ruled out by identical results seen in PANX1-Flag over-expressing cells using anti-Flag antibody for detection (Supplementary Fig. 10b).

To test whether PANX1 might be a direct target of caspases, we performed an in vitro cleavage assay by mixing purified active caspases with immunoprecipitated PANX1-Flag protein. Of those caspases tested, caspases 3 and 7 displayed the most significant cleavage of PANX1-Flag (Fig. 4d), resulting in loss of PANX1 immunoreactivity. This was not due to random activity of these caspases, as another C-terminally Flag-tagged protein, ELMO1Flag, was not cleaved in the same assay (Supplementary Fig. 10c). Caspase cleavage sites are typically tetrapeptide sequences ending in an aspartic acid, after which the cleavage occurs $^{22}$. Using the online tool CASVM ${ }^{23}$, we identified two potential caspase cleavage sites in human PANX1: DMRD in the intracellular loop (residues 164-167; denoted site A) and DVVD in the C-terminal tail (residues 376-379; denoted site B) (Fig. 4c). To detect directly the C-terminal cleavage product of PANX1 in apoptotic cells, we established Jurkat cells stably expressing PANX1 with a C-terminal green fluorescent protein (GFP) tag. Induction of apoptosis in PANX1-GFP-expressing cells resulted in a cleavage product that suggested cleavage at site B (Fig. 4e). We then mutated sites A and B (individually and together) by substituting an alanine for the aspartic acid residues (see Methods) and performed caspase cleavage reactions. Detection of cleavage products via two different antibodies (to the second extra-cellular loop (EL2) and to the C-terminal Flag tag) (Fig. If and Supplementary Fig. 10c) showed that site A and site B are indeed two target sites for caspases 3 and 7 in vitro.

To test the functional relevance of these PANX1 caspase cleavage sites in cells during apoptosis, we transiently co-transfected Jurkat cells with plasmids coding for wild-type or mutant PANX1 proteins and the fluorescent protein mCherry (marker for transfection). After inducing apoptosis, we assessed YO-PRO-1 uptake by mCherry ${ }^{+}$cells using flow cytometry (Fig. 5a). PANX1-Flag expression resulted in higher YO-PRO-1 uptake than the 
control Flag vector. Expression of the PANX1 site A mutant led to the same level of increased YO-PRO-1 uptake as the wild-type-PANX1-transfected cells, suggesting that site $\mathrm{A}$ is dispensable for PANX1 function under these conditions (Fig. 5a). However, cells expressing the PANX1 site B mutant showed a dramatic reduction in YO-PRO-1 uptake, suggesting a dominant-negative effect on YO-PRO-1 uptake by apoptotic cells (YO-PRO-1 mean fluorescence intensities of 1,393 in the site B mutant condition, versus 11,179 in the control-vector-transfected cells) (Fig. 5a). Because PANX1 is thought to function as an oligomer $^{24}$, perhaps a certain number of subunits within the complex have to be cleaved for channel activation, resulting in a dominant-negative effect due to the presence of subunits that cannot be cleaved. It is notable that PANX1-site-B-mutant-expressing cells did undergo apoptosis (annexin-V-positive). Mutation of the aspartic acid residues to glutamic acid residues (denoted site $\mathrm{B}$ EE) replicated the findings with the alanine substitutions, ruling out charge alterations as a cause (Supplementary Fig. 11a). To assess the effect of the PANX1 site B mutant on plasma membrane currents, we transiently co-transfected Jurkat cells with plasmids for a fluorescent marker and the various PANX1 constructs. Marker-positive and dying cells (actively blebbing) revealed a dramatic inhibition of apoptosis-induced, CBXsensitive current in the PANX1-site-B-mutant-expressing cells (Fig. 5b). We also established Jurkat cells stably expressing the PANX1 site B mutant. Upon apoptosis induction, ATP release and TO-PRO-3 uptake were drastically reduced (Fig. 5c, d), although the cells became apoptotic (annexin-V-positive) (Fig. 5d). These data suggest that the caspase cleavage site in the $\mathrm{C}$ terminus of PANX1 (site B) is necessary for the induction of PANX1-mediated plasma membrane permeability during apoptosis.

We next asked whether a PANX1 protein engineered to mimic truncation at site B (see Methods) would result in basal/constitutive plasma membrane permeability. Transient expression of the PANX1 truncation mutant led to TO-PRO-3 and YO-PRO-1 uptake by approximately one-third of the transfected cells (Fig. 5e and data not shown). We also detected PANX1 currents on cells expressing the truncation mutant that appeared 'live' (not blebbing), suggesting that the currents were due to expression of a constitutively open channel (Fig. 5f). The current-voltage relationship for the truncation mutant resembles that of an activated wild-type PANX1 channel, and the currents were CBX-sensitive (Fig. 5f and Supplementary Fig. 11b). Interestingly, the PANX1 currents were only seen in cells that were brightest for the co-transfected GFP marker, suggesting that only cells expressing higher amounts of the truncated/activated subunits form active channels. We performed the truncation mutant studies $16 \mathrm{~h}$ post-transfection, as these cells began to die $24-48 \mathrm{~h}$ posttransfection, perhaps due to continued release of ATP (data not shown). These data identify that cleavage of PANX1 at site B is necessary and sufficient to activate the channel, defining a new mechanism for regulation of pannexin channels.

Collectively, these data provide several new insights. This work identifies a new physiological function for PANX1, demonstrating an essential and non-redundant role in nucleotide/find-me signal release from apoptotic cells. These data have implications for how apoptotic cells may modify their extracellular microenvironment through the selective release of molecules ${ }^{25,26}$. PANX1 also contributes significantly to plasma membrane permeability during apoptosis, which is relevant for 'selective' dye uptake by early apoptotic cells. There are still limited examples where caspase-mediated cleavage of specific proteins during apoptosis subsequently translates to biologically relevant functions ${ }^{27,28}$. Our work uncovers a novel caspase-mediated mechanism of PANX1 activation during apoptosis. Since cleavage site B is evolutionarily conserved among PANX1 homologues (human, mice and zebrafish), this may be a conserved mechanism of PANX1 regulation during apoptosis. Since ATP released by dying tumour cells can activate NLRP3/inflammasome-dependent signalling and promote anti-tumour immunity ${ }^{4}$, defining a role for PANX1 in ATP release could also have implications for targeting these channels in cancer therapies. 


\section{METHODS SUMMARY \\ Induction of apoptosis}

Jurkat cells resuspended in serum-free media (RPMI/1\% BSA) were treated with $250 \mathrm{ng}$ $\mathrm{ml}^{-1}$ anti-Fas (CH11 clone) or $100 \mathrm{~mJ} \mathrm{~cm}^{-2}$ ultraviolet C irradiation (Stratalinker). Primary murine thymocytes were treated with cross-linked anti-Fas $\left(5 \mu \mathrm{g} \mathrm{ml}^{-1}\right.$ anti-Fas (Jo2 clone) + $2 \mu \mathrm{g} \mathrm{ml}^{-1}$ protein $\left.\mathrm{G}\right)$.

\section{Preparation of cell supernatants and nucleotide measurement}

Cells were incubated for indicated times after receiving treatments. Supernatants were collected by two consecutive centrifugations ( $425 \mathrm{~g}$ for $2 \mathrm{~min}$ at room temperature). ATP was measured using a luciferase/luciferin assay (CellTiter-Glo; Promega) according to manufacturer's instructions. UTP concentrations were quantified by the UDP-glucose pyrophosphorylase-based reaction, as described previously ${ }^{29}$.

\section{Migration assay}

Transwell migration assays were performed by applying THP-1 cells to the insert and the chemoattractant in the well of a 24-well plate with 5- $\mu \mathrm{m}$ pore Transwells (Corning) for $1 \mathrm{~h}$ at $37^{\circ} \mathrm{C}$. Supernatants were diluted in some cases to bring the chemotactic activity into the linear range of the migration assay, with the same dilution applied to all samples in each experiment. Percentage of input cells that migrated to the lower chamber was determined by flow cytometry using AccuCount beads (Spherotech).

\section{PANX1 constructs}

Human PANX1-Flag construct was generated by PCR cloning of PANX1 cDNA (Open Biosystems; catalogue number MHS1010-58324) into pEBB-Flag vector. Cleavage site mutations were performed using a QuikChange mutagenesis kit (Stratagene). PANX1 truncation mutant (amino acids 1-371) was produced by PCR to end at amino acid 371, immediately followed by a Flag tag (DYKDDDDK), resulting in a 379 amino acid protein (size that would be produced by caspase cleavage after the last aspartic acid residue in site B).

\section{Patch-clamp analysis}

Whole-cell recordings were obtained at room temperature using patch pipettes (3-5 M $\Omega$ ) filled with a Cs-based internal solution. Peak current $($ at $+90 \mathrm{mV})$ was normalized to cell capacitance. CBX-sensitive current was taken as the difference in peak current before and after CBX application.

\section{Supplementary Material}

Refer to Web version on PubMed Central for supplementary material.

\section{Acknowledgments}

We thank members of the Ravichandran laboratory for their comments and suggestions. We also thank R. E. Campbell for providing the construct for mBlueberry2. This work was supported by the Pharmacological Sciences Training Grant (F.B.C. and J.K.S.) (NIGMS), a F30 pre-doctoral fellowship from the NHLBI (F.B.C.), a predoctoral fellowship from the AHA (J.K.S.), and grants from the National Institutes of Health (K.S.R.). J.M.K. is supported by grants from the American Heart Association (Scientist Development Grant) and the American Cancer Society. K.S.R. is a William Benter Senior Fellow of the American Asthma Foundation. 


\section{References}

1. Lauber K, Blumanthal SC, Waibel M, Wesselborg S. Clearance of apoptotic cells: getting rid of the corpses. Mol Cell. 2004; 14:277-287. [PubMed: 15125832]

2. Elliott MR, et al. Nucleotides released by apoptotic cells act as a find-me signal to promote phagocytic clearance. Nature. 2009; 461:282-286. [PubMed: 19741708]

3. Idziorek T, Estaquier J, De Bels F, Ameisen JC. YOPRO-1 permits cytofluorometric analysis of programmed cell death (apoptosis) without interfering with cell viability. J Immunol Methods. 1995; 185:249-258. [PubMed: 7561136]

4. Ghiringhelli F, et al. Activation of the NLRP3 inflammasome in dendritic cells induces IL-1 $\beta$ dependent adaptive immunity against tumors. Nature Med. 2009; 15:1170-1178. [PubMed: 19767732]

5. Fujiwara T, Oda K, Yokota S, Takatsuki A, Ikehara Y. Brefeldin A causes disassembly of the Golgi complex and accumulation of secretory proteins in the endoplasmic reticulum. J Biol Chem. 1988; 263:18545-18552. [PubMed: 3192548]

6. Sebbagh M, et al. Caspase-3-mediated cleavage of ROCK I induces MLC phosphorylation and apoptotic membrane blebbing. Nature Cell Biol. 2001; 3:346-352. [PubMed: 11283607]

7. Coleman ML, et al. Membrane blebbing during apoptosis results from caspase-mediated activation of ROCK I. Nature Cell Biol. 2001; 3:339-345. [PubMed: 11283606]

8. Harris AL. Connexin channel permeability to cytoplasmic molecules. Prog Biophys Mol Biol. 2007; 94:120-143. [PubMed: 17470375]

9. MacVicar BA, Thompson RJ. Non-junction functions of pannexin-1 channels. Trends Neurosci. 2010; 33:93-102. [PubMed: 20022389]

10. Scemes E, Spray DC, Meda P. Connexins, pannexins, innexins: novel roles of "hemi-channels". Pflugers Arch. 2009; 457:1207-1226. [PubMed: 18853183]

11. Ma W, Hui H, Pelegrin P, Surprenant A. Pharmacological characterization of pannexin-1 currents expressed in mammalian cells. J Pharmacol Exp Ther. 2009; 328:409-418. [PubMed: 19023039]

12. Denault JB, Salvesen GS. Apoptotic caspase activation and activity. Methods Mol Biol. 2008; 414:191-220. [PubMed: 18175821]

13. Silverman W, Locovei S, Dahl G. Probenecid, a gout remedy, inhibits pannexin 1 channels. Am J Physiol Cell Physiol. 2008; 295:C761-C767. [PubMed: 18596212]

14. Bruzzone R, Barbe MT, Jakob NJ, Monyer H. Pharmacological properties of homomeric and heteromeric pannexin hemichannels expressed in Xenopus oocytes. J Neurochem. 2005; 92:10331043. [PubMed: 15715654]

15. Baranova A, et al. The mammalian pannexin family is homologous to the invertebrate innexin gap junction proteins. Genomics. 2004; 83:706-716. [PubMed: 15028292]

16. Söhl G, Maxeiner S, Willecke K. Expression and functions of neuronal gap junctions. Nature Rev Neurosci. 2005; 6:191-200. [PubMed: 15738956]

17. Locovei S, Scemes E, Qiu F, Spray DC, Dahl G. Pannexin1 is part of the pore forming unit of the $\mathrm{P} 2 \mathrm{X}_{7}$ receptor death complex. FEBS Lett. 2007; 581:483-488. [PubMed: 17240370]

18. Chen X, Shu S, Kennedy DP, Willcox SC, Bayliss DA. Subunit-specific effects of isoflurane on neuronal Ih in HCN1 knockout mice. J Neurophysiol. 2009; 101:129-140. [PubMed: 18971302]

19. Thompson RJ, et al. Activation of pannexin-1 hemichannels augments aberrant bursting in the hippocampus. Science. 2008; 322:1555-1559. [PubMed: 19056988]

20. Spencer SL, Gaudet S, Albeck JG, Burke JM, Sorger PK. Non-genetic origins of cell-to-cell variability in TRAIL-induced apoptosis. Nature. 2009; 459:428-432. [PubMed: 19363473]

21. Penuela $S$, et al. Pannexin 1 and pannexin 3 are glycoproteins that exhibit many distinct characteristics from the connexin family of gap junction proteins. J Cell Sci. 2007; 120:37723783. [PubMed: 17925379]

22. Pop C, Salvesen GS. Human caspases: activation, specificity, and regulation. J Biol Chem. 2009; 284:21777-21781. [PubMed: 19473994]

23. Wee LJ, Tan TW, Ranganathan S. CASVM: web server for SVM-based prediction of caspase substrates cleavage sites. Bioinformatics. 2007; 23:3241-3243. [PubMed: 17599937] 
24. Ambrosi C, et al. Pannexin1 and pannexin2 channels show quaternary similarities to connexons and different oligomerization numbers from each other. J Biol Chem. 2004; 285:24420-24431. [PubMed: 20516070]

25. Burnstock G, Knight GE. Cellular distribution and functions of P2 receptor subtypes in different systems. Int Rev Cytol. 2004; 240:31-304. [PubMed: 15548415]

26. Praetorius HA, Leipziger J. ATP release from non-excitable cells. Purinergic Signal. 2009; 5:433446. [PubMed: 19301146]

27. Johnson CE, Kornbluth S. Caspase cleavage is not for everyone. Cell. 2008; 134:720-721. [PubMed: 18775303]

28. Timmer JC, Salvesen GS. Caspase substrates. Cell Death Differ. 2007; 14:66-72. [PubMed: 17082814]

29. Lazarowski ER, Harden TK. Quantitation of extracellular UTP using a sensitive enzymatic assay. Br J Pharmacol. 1999; 127:1272-1278. [PubMed: 10455275] 


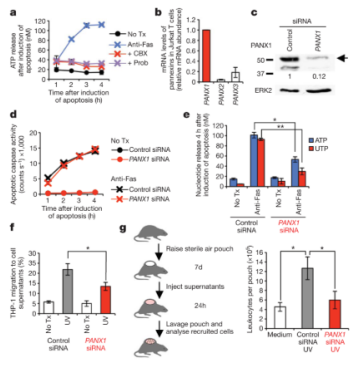

Figure 1. Release of find-me signals by apoptotic cells is pannexin-1-dependent

a, Time course of ATP release from apoptotic Jurkat cells in the presence of $500 \mu \mathrm{M}$ carbenoxolone (CBX) or $2 \mathrm{mM}$ probenecid (Prob). $n=3$. No Tx, no treatment. b, mRNA levels for $P A N X 1, P A N X 2$ and $P A N X 3$ in Jurkat cells determined by qPCR, normalized to $P A N X 1 . n=2$. c, PANX1 protein expression in Jurkat cells transfected with control or PANX1 siRNA. Glycosylated PANX1 (upper band; arrow) was quantified. d, e, PANX1 knockdown does not affect the progression of apoptosis (assessed by apoptotic caspase activity) (d, $n=3)$, but decreases ATP $(\mathbf{e}, n=10)$ and UTP $(\mathbf{e}, n=3)$ release $4 \mathrm{~h}$ after apoptosis induction. *, $P<10^{-5} ; * *, P<0.01$. f, Transwell migration of THP-1 monocytes towards apoptotic cell supernatants from PANXI-siRNA-treated cells ( $4 \mathrm{~h}$ after ultraviolet (UV) treatment). ${ }^{*}, P<0.05$. Representative of four independent experiments. $\mathrm{g}$, Left, Schematic of mouse air pouch model for monitoring chemotactic activity of apoptotic cell supernatants in vivo. Right, $\mathrm{CD} 45^{+}$leukocytes migrating into the pouch were determined after injecting apoptotic cell supernatants from siRNA-transfected cells. $n=9-10$ mice per group. * $P<0.05$, by ANOVA with Bonferroni post-analysis. Error bars, s.e.m., except in b, f, where they represent s.d. 

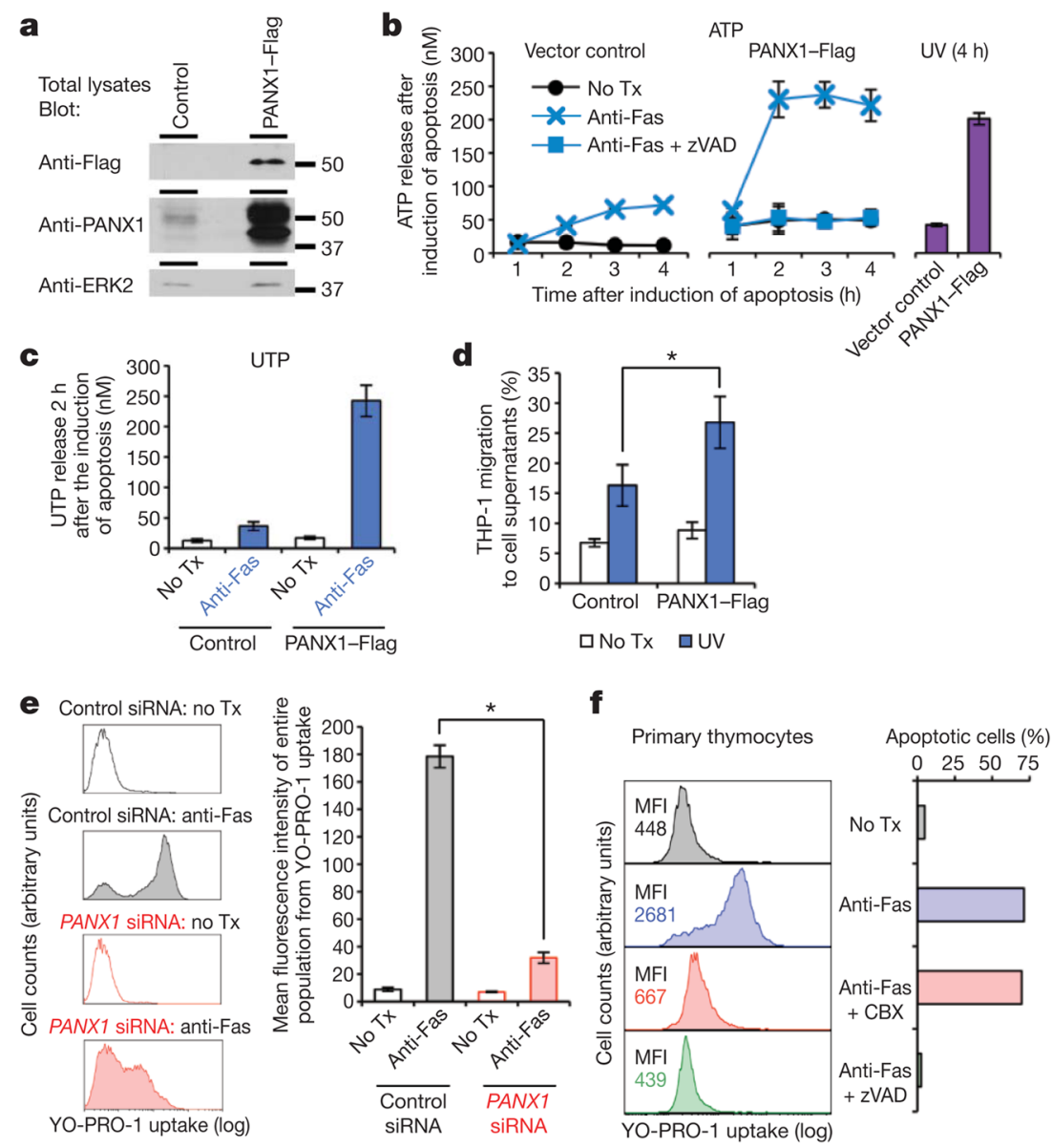

Figure 2. Pannexin 1 expression level correlates with find-me signal release and membrane permeability

a, Immunoblotting of lysates from control or PANX1-Flag Jurkat cells. b, ATP release by Jurkat cells after induction of Fas-mediated apoptosis, and $4 \mathrm{~h}$ after UV-induced apoptosis. $n$ $=2$. $\mathbf{c}$, UTP levels in supernatants of Jurkat cells $2 \mathrm{~h}$ after apoptosis induction. $n=4$. d, Migration of THP-1 monocytes to supernatants from control and PANX1-Flag expressing cells, collected $2 \mathrm{~h}$ after induction of apoptosis. $*, P<0.05$. Representative of four experiments. e, Left, flow cytometry histograms showing YO-PRO-1 dye uptake by control versus PANX1-siRNA-transfected Jurkat cells with or without apoptosis induction. Right, uptake of YO-PRO-1 presented as mean fluorescence intensity (MFI) of the entire cell population. *, $P<0.0005, n=3$. f, Left, YO-PRO- 1 uptake by primary murine thymocytes undergoing anti-Fas-induced apoptosis, with zVAD or CBX treatments. Right, percentage of apoptotic cells (annexin $\mathrm{V}$ positive and propidium iodide negative). $n=2$. Error bars represent s.d., except in c (s.e.m.). 


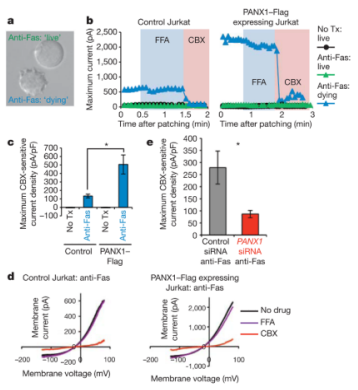

Figure 3. Carbenoxolone-sensitive current induced during apoptosis is pannexin-1-dependent a, Morphology of anti-Fas treated Jurkat cells used to identify 'live' and 'dying' cells (based on blebbing). b, Patch-clamp recordings from Jurkat cells, receiving indicated treatments. Peak whole-cell current (at $+90 \mathrm{mV}$ ) is shown under conditions when bath solution was perfused with FFA (100 $\mu \mathrm{M}$, blue shading) or CBX (100 $\mu \mathrm{M}$, pink shading). Exemplar traces are representative of 5-15 cells per group. c, CBX-sensitive current density in indicated Jurkat cells, normalized to whole-cell capacitance ( $\mathrm{pA} / \mathrm{pF})$. ${ }^{*}, P<0.01 . n \geq 7$ per group. d, Current-voltage relationships of apoptosis-induced current in dying Jurkat cells (from b), with current measured over a range of voltages (only dying cells shown, as live cells have very little current). Note different $y$ axes. Traces representative of $4-15$ cells. e, CBX-sensitive current density in PANX1-siRNA-transfected cells induced to undergo apoptosis. *, $P<0.05 . n \geq 8$ per group. All error bars represent s.e.m. 


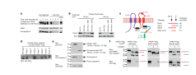

Figure 4. Pannexin 1 is a target of effector caspase cleavage during apoptosis

a, b, Fas-mediated apoptosis results in loss of PANX1 detection with antibody targeted to PANX1 C terminus in Jurkat cells (a) and primary murine thymocytes (b). c, Schematic of PANX1-Flag protein indicating the predicted caspase cleavage sites (sites A and B) and the epitopes recognized by the EL2 (extracellular loop 2) and C-terminal antibodies. The predicted products of caspase cleavage at sites A and B are also shown. d, In vitro cleavage of immunoprecipitated PANX1-Flag incubated with the indicated purified active caspases. Loss of immunoreactivity to Flag assessed. Representative of two independent experiments. e, Jurkat cells stably expressing a C-terminally GFP-tagged PANX1 were induced to undergo apoptosis and assessed for the cleaved C-terminal fragment (which runs at $\sim 33$ $\mathrm{kDa}$ ). Representative of two independent experiments. $\mathbf{f}$, In vitro cleavage of indicated PANX1-Flag proteins (wild type and caspase cleavage site mutants), incubated with the indicated caspases. 


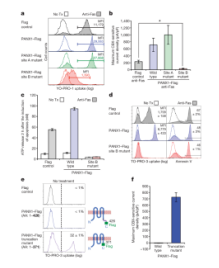

Figure 5. Caspase-mediated cleavage of pannexin 1 results in channel activation during apoptosis

a, b, Jurkat cells transiently transfected with indicated constructs were assessed for YOPRO-1 uptake (a) and current density (b) $48 \mathrm{~h}$ post-transfection. The MFI for YO-PRO-1 uptake by the gated population is shown (a) and the analysis of dying cells in $\mathbf{b}$ was performed as in Fig. 3. *, $P<0.01, n \geq 5$ for each group. c,d, Jurkat cells stably transfected with the indicated plasmids were assessed for ATP release (c, $n=3)$, TO-PRO-3 uptake (d, left) and annexin $\mathrm{V}$ staining (d, right) $2 \mathrm{~h}$ after induction of apoptosis. MFI for the TOPRO-3 positive peaks and percentage of annexin $\mathrm{V}$ positive cells are shown. $n=3$. e, TOPRO-3 uptake by Jurkat cells $16 \mathrm{~h}$ after transient transfection with full-length or truncated PANX1. Schematic of full-length and truncated PANX1 (amino acids 1-426 and 1-371, respectively) are shown. $n=3$. f, CBX-sensitive currents in Jurkat cells transiently transfected with indicated PANX1 proteins (full length or truncation mutant). Patch-clamp was performed on cells that appeared morphologically healthy. $n \geq 5$ per group. Error bars and \pm represent s.e.m. 